\title{
MEASUREMENT OF BEAM CHARACTERISTICS OF PLS LINAC*
}

\author{
J.-Y. Choï, H. S. Kang, S. H. Nam, S. S. Chang \\ Pohang Accelerator Laboratory, POSTECH, Pohang 790-784, Korea
}

\begin{abstract}
The PLS (Pohang Light Source) linac has been operated routinely as the injection linac for PLS, a thirdgeneration synchrotron light source. The transverse electron beam emittance was measured at the preinjector end of the PLS linac, where the beam energy is about $100 \mathrm{MeV}$. The emittance was measured using a quadrupole magnet and a fluorescent target. We used a progressive scan CCD camera with an asynchronous random trigger shutter to obtain precise beam sizes. The beam image analysis was made with a PC equipped with a frame grabber. Bunch length was also measured at the pre-injector end using a streak camera to observe transition radiation emitted from a radiator inserted into the beam path. The measurement system and the results are described in detail.
\end{abstract}

\section{INTRODUCTION}

The PLS (Pohang Light Source) linac has been operated as the full energy injection linac for the PLS, a third generation synchrotron light source storage ring [1,2]. It provides electron beams to the PLS storage ring twice a day. In the regular injection mode, the electron beams from the gun has a pulse width of less than 1 ns (FWHM), beam current of $1.7 \mathrm{~A}$, and repetition rate of $10 \mathrm{~Hz}$.

Just after the completion of the linac and during the commissioning period in 1994, beam parameters were measured and reported [1]. To further improve the beam quality and the operation stability of the linac, we are again measuring the electron beam parameters at the linac.

In this paper we describe some preliminary results on the emittance and bunch length measurements done at the pre-injector of the linac. The pre-injector includes the bunching system and two accelerating columns. The prebuncher is an S-band standing-wave type cavity, and the buncher has a traveling-wave structure with four cavities. The pre-injector is powered by the first klystron-modulator module, and accelerates the electron beam to $100 \mathrm{MeV}$.

Figure 1 shows typical electron beam transmission efficiency along the linac, under the usual beam acceleration condition for normal injection into the

"Work supported by Ministry of Science and Technology and POSCO

"Email: choij@postech.ac.kr storage ring. The first wall current monitor (WCM) is located near the exit of the electron gun and the second WCM is located at the end of pre-injector. The electron beam loss occurs mainly during the bunching process and the first acceleration stage, and the transmission efficiency at the pre-injector is about $60 \%$. After the preinjector, the electron beam is transmitted to the linac end with small beam loss. The results reported in this paper refer to this beam condition unless otherwise stated.

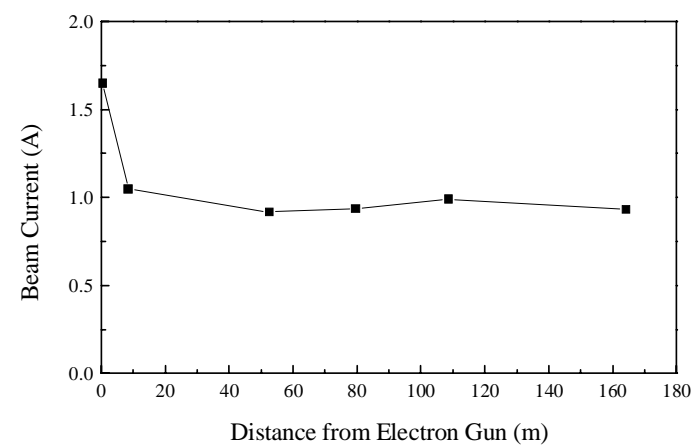

Figure 1: Beam current transmission efficiency along the linac measured by wall current monitors.

\section{EMITTANCE MEASUREMENT}

The transverse electron beam emittance was measured at the pre-injector using a quadrupole magnet $(\mathrm{QM})$ and a beam profile monitor (BPRM) utilizing a fluorescent screen.

In order to measure the exact electron beam spot sizes on the BPRM screen, we used a frame grabber (LBA300PC, Spiricon Inc.) and an asynchronous CCD camera (CV-M10, Costar Ind.), in which the camera shutter timing can be controlled by the beam trigger. To have a good resolution the camera was operated in the progressive scan mode during the measurements.

Although we have one set of a QM and a BPRM in the drift space of the pre-injector, the distance between the QM and the BPRM is too short to observe an appreciable change of the beam spot size with the change of the QM excitation current. Instead, we measured the beam size at a BPRM located in the beam energy analyzing station. Since the horizontal beam size can be affected by the dispersion function of the bending magnet, it is 
necessary to isolate its contribution from the observed horizontal beam size.

The beam spot sizes in the vertical direction are shown in the Fig. 2 as a function of the QM excitation current. The emittance was calculated from these measured beam sizes by the relationship on the beam radius and beam matrix elements [3]. The calculated emittance value was about $80 \pi \cdot \mathrm{mm} \cdot \mathrm{mrad}$. While the beam intensity profiles were Gaussian shapes in the vertical direction, the horizontal beam sizes varied largely from pulse to pulse. Therefore, no reliable data about beam spot sizes were obtained in the horizontal direction during the preliminary measurement. This pulse-to-pulse beam size variation is believed to be caused by beam energy jitters, probably due to the temporal instability of the modulator and other components. We are planning to continue the emittance measurement at the pre-injector after reducing the energy jitters.

The energy spread of the electron beams at the preinjector was measured from the horizontal beam sizes in the beam analyzing station using the frame grabber. The observed energy spread at the pre-injector was about $\pm 0.27 \%$, and this energy spread varied from \pm 0.24 to $\pm 0.3 \%$ from pulse to pulse.

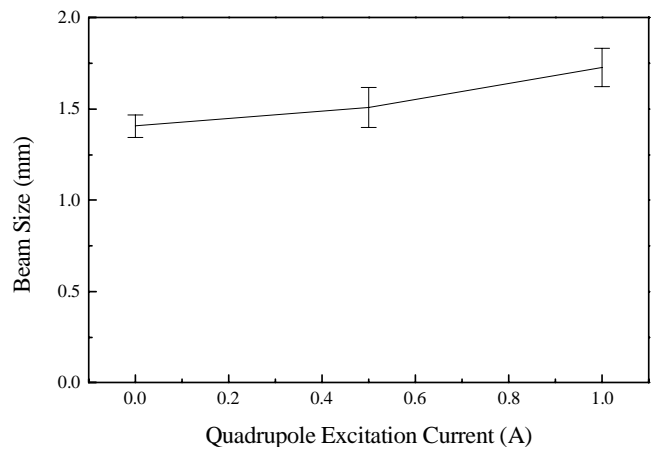

Figure 2: Variation of the vertical beam spot size with the current of the focusing quadrupole magnet.

\section{BUNCH LENGTH MEASUREMENT}

Bunch lengths were measured using the optical transition radiation (OTR) emitted from a metal target when accelerated electron beams impinged on the target. The bunch monitor system utilizing OTR has favorable characteristics to accurately measure the bunch shape and the bunch length, and is widely used in electron linacs $[4,5]$. The schematic layout of the measurement system is shown in Fig. 3.

The target is made of stainless steel $2.6 \mathrm{~mm}$ thick, and its surface was polished to the roughness less than 0.1 $\mu \mathrm{m}$ in Ra value. The target chamber was installed at the pre-injector end where the electron energy is $100 \mathrm{MeV}$. The emitted OTR is guided to the gallery via optical components such as lenses and mirrors. To block stray light and to have better $\mathrm{S} / \mathrm{N}$ ratio, the optical components were surrounded by a light-shielding structure. The guided OTR was measured by a streak camera (Hamamatsu Photonics K. K. Co.) with a fast single sweep unit. Since the beam intensity was not high enough, the alignment of the optical components was critical in the measurement. We aligned the optical components with a He-Ne laser. The optical path from the target to the streak camera is about $10 \mathrm{~m}$ long. The time resolution of the streak camera is less than $2 \mathrm{ps}$, which is a sufficiently small value for S-band linac bunch length measurement. To keep better time resolution the slit width of the streak camera was kept as narrow as $20 \mu \mathrm{m}$.

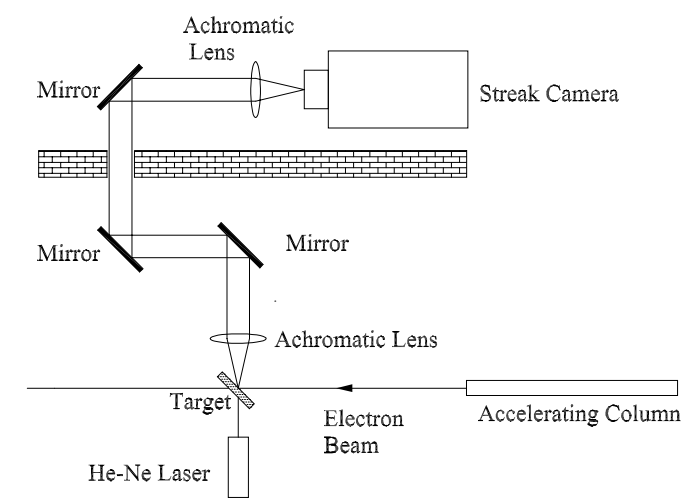

Figure 3: Layout of the bunch length measurement system.

Figure 4 shows two examples of the streak camera image of the bunch train contained in one electron beam pulse. The electron beam has a pulse width less than $1 \mathrm{~ns}$ (FWHM) and a beam current of about $1 \mathrm{~A}$. Five bunches are observed in one beam pulse.
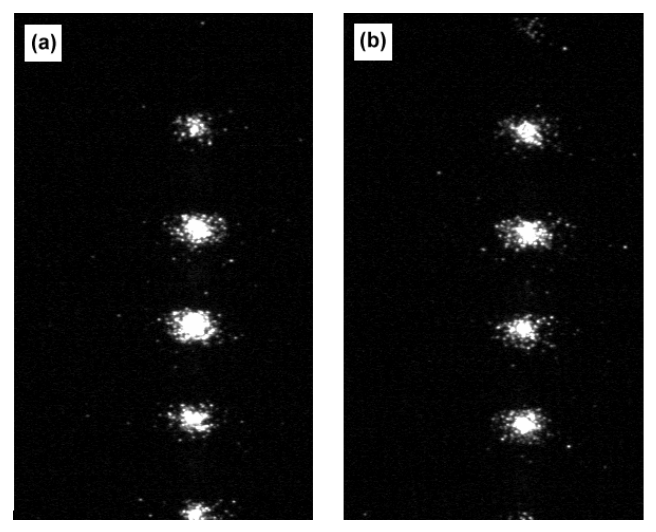

Figure 4: Streak camera images of the bunch train contained in one beam pulse of 1 ns. The vertical direction corresponds to time axis and in this case time range is $2 \mathrm{~ns}$.

As is clear in Fig. 4(a), the bunch lengths were different from bunch to bunch. The length of a bunch 
with more charge content tends to be larger. In addition, the charge distributions among the bunches show different pattern from pulse to pulse. On the other hand, Fig. 4(b) shows relatively uniform bunch lengths. These phenomena can be explained qualitatively by two facts; the time structure of the electron charge emitted from the electron gun has a triangular shape due to a short pulse length, and the electron beam and RF phase of the bunching system do not have definite synchronization in our linac.

The effects of RF parameters of the bunching system on bunch length have been investigated. Measurements were done for the beam of 2-ns pulse length to reduce the above-mentioned bunch length variation from bunch to bunch and from pulse to pulse. Figure 5 shows the bunch image and intensity profile for the case where RF parameters of the bunching system were not optimally adjusted. The bunch lengths in this case were more than 17 ps. On the other hand, Fig. 6 shows the bunch image and intensity profile after RF parameters were adjusted. Bunch lengths were reduced to less than 12 ps by the adjustment of the RF parameters of the bunching system.
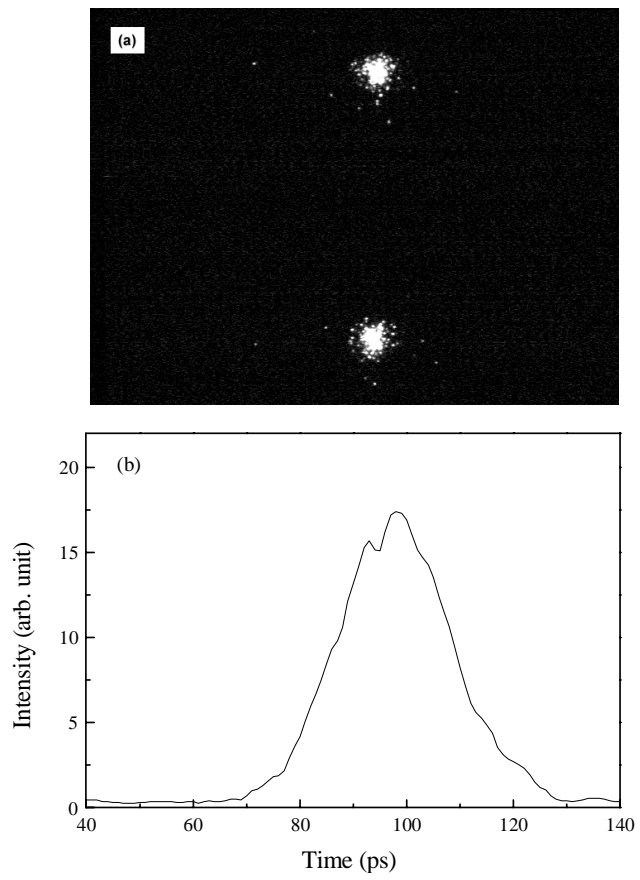

Figure 5: (a) Bunch image and (b) intensity profile of bunch before RF parameters of the bunching system were adjusted. In (a) the vertical axis corresponds to streak time whose range is $500 \mathrm{ps}$, and the intensity profile of the lower bunch is plotted in (b).

\section{SUMMARY}

We have been measuring electron beam parameters at the pre-injector of the PLS linac. The measured beams have the same condition as the beam injected to the
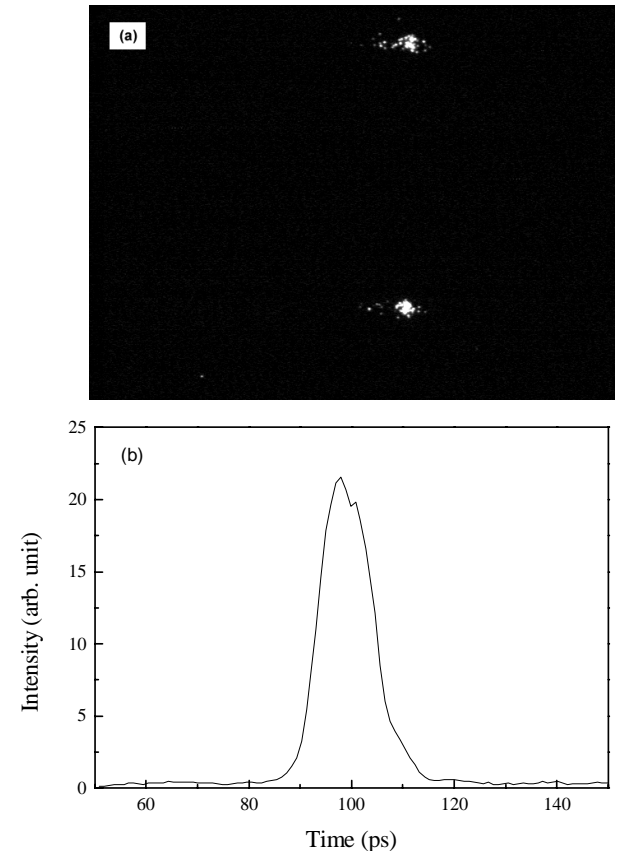

Figure 6: (a) Bunch image and (b) intensity profile of the bunch after RF parameters of the bunching system were adjusted. The intensity profile represents the lower bunch shown in (a).

storage ring. The transverse emittance in the vertical direction was measured. Preliminary measurement results were presented. We are planning to continue the bunch length and emittance measurement at the preinjector after reducing the pulse-to-pulse energy jitters. The beam parameter measurements at the linac end are now in progress.

\section{REFERENCES}

[1] W. Namkung, "PLS 2.0-GeV LINAC", Proceedings of the 1994 International Linac Conference, August 21-26, 1994, Tsukuba, Japan

[2] S. H. Nam, K. R. Kim, Y. J. Han, Y. C. Kim, and S. S. Chang, "Status of PLS 2-GeV Electron Linac", in these proceedings

[3] H. Wiedemann "Particle Accelerator Physics", p. 157, SpringerVerlag (1993)

[4] M. Jablonka, J. Leroy, X. Hanus and L. Wartski, "Beam diagnostic using transition radiation produced by a $100 \mathrm{MeV}$ electron beam", DPhn Saclay 91-32, 1991.

[5] Y. Ogawa, J.-Y. Choi, T. Suwada, T. Kamitani, T. Urano, K. Furukawa, S. Ohsawa, A. Enomoto and I. Sato, "Beam Monitor Utilizing Transition Radiation" 1993 Proceedings of the 1993 Particle Accelerator Conference, May 17-20, Washington, D. C., USA, 1993 